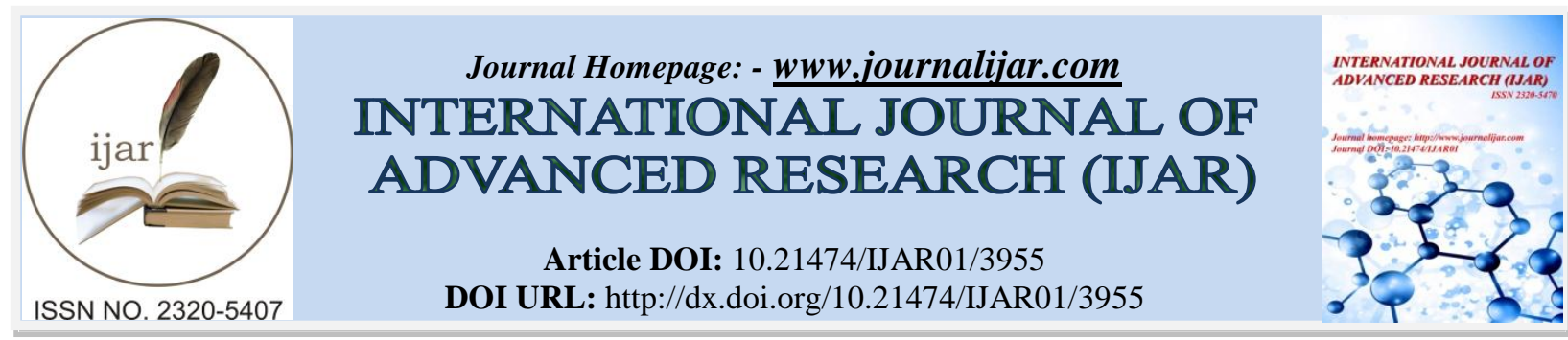

RESEARCH ARTICLE

\title{
A REVIEW ON IMAGE REGISTRATION ON PRINCIPLE AXES METHOD AND MUTUAL INFORMATION METHOD.
}

Madhurima Banerjee $^{1}$ and Prof. Samir Kumar Bandyopadhyay ${ }^{2}$.

1. Dept. of Computer Science and Application, Heritage Academy, Kolkata, India.

2. Dept. of Computer Sc. \& Engineering, University of Calcutta, Kolkata, India.

\section{Manuscript Info}

Manuscript History

Received: 03 February 2017

Final Accepted: 06 March 2017

Published: April 2017

Key words:-

Image registration, Feature detection,

Feature matching and Mapping function

\begin{abstract}
Image registration is an image processing technique, where different images are considered and the images are aligned together using some reference points, so that additional information can be inferred from the images. Several algorithms have been developed for registration. Fourier Transformation method, interpolation method, principal axes method, mutual information method are some of those algorithms. This paper deals mainly with rigid image transformation. The Principle Axes method and Mutual Information method of image registration have been reviewed.
\end{abstract}

Copy Right, IJAR, 2017,. All rights reserved.

\section{Introduction:-}

Image registration considers more than one image of the same object, taken either from different angles, or by using different means, or at different temporal dimension. One image may project partial section of another image. These images which differ in angular or spatial or temporal dimension is aligned together in image registration process to gather more information either through comparative analysis or through composite analysis of the resultant image. Aligning the images is one of the major hurdle in image registration process.

Lisa Tang and GhassanHamarneh[6] have defined image registration as:

Let there be two images A and B. The goal of registering A and B is to find a spatial transformation $\mathrm{U}$ that optimally maps points in Ato the corresponding points in $\mathrm{B}[6]$.

$\mathrm{U}: \mathrm{xA} \rightarrow \mathrm{xB} \Leftrightarrow \mathrm{U}(\mathrm{xA})=\mathrm{xB}$.

As per Zitova and Flusser [4], image registration process mainly consists of following four steps:

Feature detection $\rightarrow$ Feature Matching $\rightarrow$ Transform model estimation $\rightarrow$ Image resampling and transformation. During image registration, it is important to decide what kind of features of the two images are needed to be mapped.The features detected can be feature based or area based. The features thus detected must have common elements in the two images for the purpose of registration [4-5].

While mapping the two images for registration, the concept of image degradation should be considered. Degradation can arise due to artifacts or due to different techniques used to capture the images.

One of the challenges in image registration is distortion, i.e., coordinate (xa,ya) in the source image A may not correspond to the same coordinate (xb,yb) inthe target image B. Derek L G Hill, Philipp G Batchelor, Mark Holden and David J Hawkes [7], have discussed about geometrical distortions where they have stated that theoretically 
geometrical distortions can be corrected, however, in practice, it may not be feasible. Scaling, skewing, pin cushion, barrel distortions are some of the geometrical distortions.

Therefore one of the first steps in image registration is to check whether the images are misaligned or distorted with respect to each other.

If the images are aligned with respect to each other, then mapping the corresponding coordinates in the two images becomes very easy, straight forward and simple. Whereas, if the images are found to be distorted with respect to each other, a technique has to be realized to map the two images, such that the two images synchronize with each other. The common aim of all the algorithms used for the mapping purpose, is to find a relationship or function between (xa,ya) of the source image A and (xb,yb) of the target image B where (xa,ya) and (xb,yb) are the corresponding points in the two images.

Once the mapping function has been identified, the images can be aligned using image transformation, the simplest of the transformations are translation and rotation.Image mapping techniques can be broadly categorized into rigid and non-rigid techniques.

Considering medical images, they are used to diagnosis, plan treatment and monitor state of disease, especially in neuroscience. Such images are acquired from patients at different times, and often have different modalities. Considering medical images, MRI, CT and PET scans can be aligned together to extract additional information from the images. Image registration can include 2D-2D, 3D-3D or 2D-3D registrations.

Registration of brain images were the earliest usage of image registration. One of the most common use of image registration of the brain is to track growth of tumor, but with proper image registration, any change in the brain area can be detected.

Shape of the brain does not change much. It can be considered as an elliptic geometrical shape and that position of the brain inside the skull remains same, these facts makerigid image registration techniqueone of the registration techniques for brain image analysis.Rigid or linear image registration technique involves less than ten independent dimensions.

As Neha and Naresh has stated in their work [13], rigid transformations are defined as geometric transformations that preserve distances, straightness of lines and angles. These are global transformations and cannot handle local deformations. Rigid transformation mainly uses translation and rotation.

A Rigid body, can be located uniquely using the following knowledge [1]:

Centre of the mass

Orientation of the image with center of the mass

Principal axes registration is one of the rigid registration techniques used for brain.

Principal Axes Registration:-

For any rigid body, center of mass, inertia matrix and principal axes can be determined quite easily [1], using which, rotation and translation technique can be used to register the two images.

Principal Axes registration is a feature based registration method, where the aim is to align the corresponding points in the two images, i.e., for two images A, B

$\{x i A\} i=1 \ldots N$ in image $A$ and $\{x i B\} i=1 \ldots N$, are the corresponding features in the two images.

Principal axes coincides with axes of symmetry, which forms an orthogonal coordinate system, with origin at the center of mass. It is assumed that the brain does not deform or move within the cranium [1].

Theory:

Two images with equal enclosed volumes are considered. It is understood that if the images represent the same object, then the center of mass of both the images would coincide. Formula for center of mass can be calculated [12]. 


$$
\begin{gathered}
\hat{x}=\frac{\sum_{x, y} x I(x, y)}{\sum_{x, y} I(x, y)} \\
\hat{y}=\frac{\sum_{x, y} y I(x, y)}{\sum_{x, y} I(x, y)} .
\end{gathered}
$$

Where, Iis 2-D array representing an image,

$\mathrm{I}(\mathrm{x} ; \mathrm{y})$ represents pixel intensity at coordinate $(\mathrm{x}, \mathrm{y})$

The covariance matrix of the two images are formed. Covariance gives us the measure as to how much each of the dimensions vary from the mean with respect to each other and it determines the relationship between the two dimensions. The covariance matrix formed is a $2 \times 2$ matrix as follows[2] :

$\mathrm{C}=\left(\begin{array}{ll}\mathrm{C} 11 & \mathrm{C} 12 \\ \mathrm{C} 21 & \mathrm{C} 22\end{array}\right)$

Where,

$$
\begin{aligned}
& c_{11}=\sum_{x, y}(x-\hat{x})^{2} I(x, y), \\
& c_{22}=\sum_{x, y}(y-\hat{y})^{2} I(x, y), \\
& c_{12}=\sum_{x, y}(x-\hat{x})(y-\hat{y}) I(x, y), \\
& c_{21}=c_{12} .
\end{aligned}
$$

The eigenvectors of $\mathrm{C}$ gives the direction of the major and minor axes of the brain which has an elliptical shape.Theoretically, principal axes technique may not be applicable on objects having high degree of symmetry, cause the eigenvectors so obtained, may not be unique[3]. Practically, such high degree of symmetry is rare.

$$
\begin{gathered}
\text { C. } \vec{v}=\lambda \cdot \vec{v} \\
\vec{v}(C-\lambda I)=0
\end{gathered}
$$

Assuming that vector is not null vector, the above equation is valid if, $\operatorname{Det}(\mathrm{C}-\lambda \mathrm{I})=0$ (eq 1)

On solving equation 1, we get two eigenvalues. The two eigenvalues yield 2 eigenvectors. Smaller eigenvalue gives the direction of minor axes. Larger eigenvalue gives direction of the major axes [2].

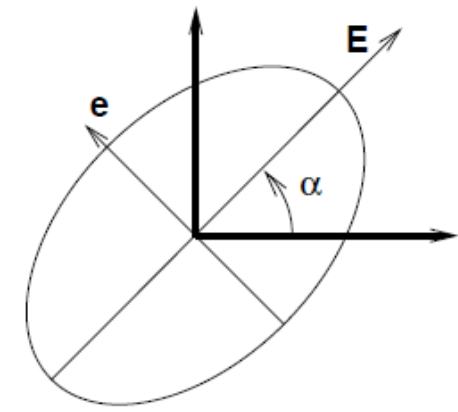

In the above diagram [1], E gives direction of major axes and e gives direction of minor axes. $\alpha$ is the angle the major axes makes with the axes parallel to the horizontal plane. 
Angle $\alpha$ and $\alpha^{\prime}$ is calculated for source and target images respectively. For the purpose of image registration, target image is rotated by an angle $\left|\alpha-\alpha^{\prime}\right|$.

Principal axes technique may not accurately align those image sections which are farther from center of mass, because principal axes for those sections may not overlap. Secondly, if the source and target images of the brain differs in shape or size then there lies a probability that principal axes technique may give rise to misalignment [3].

\section{Mutual Information Technique of Image Registration:-}

Hill et al. [9] implemented a method to check whether the two images are mis-aligned. The algorithm considers the registration technique introduced by Woods et al.[8] for multimodality images in 1993. The assumption Woods used was that regions of similar tissues which has the same intensity values in the source image would also have corresponding intensity regions in the target image, though the intensities for the same tissue type in the two images taken from different sources may vary, yet the ratios between intensities of the same tissue types will be small when the identical images are registered.

Hill in their method has created a feature space using the intensity values of the two images. The theory of the algorithm is that when the images are correctly registered, corresponding points in the images overlap and histogram created using the intensities shows certain clusters for the grey values of those structures. If the points in brain do not overlap, then, the clusters will get dispersed [10]. It is shown in figure 1.

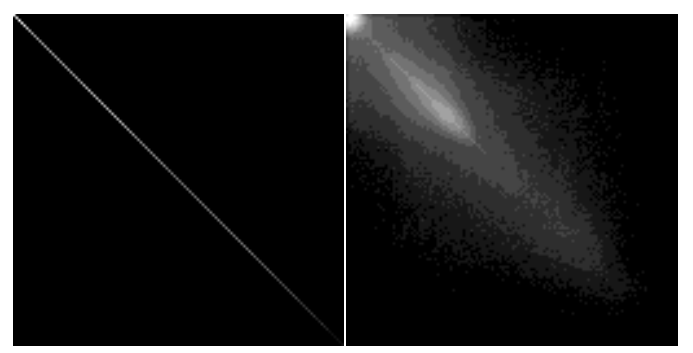

Figure 1:- Dispersed Cluster

In [10], Pluim et al has given the above two images to show that for images that overlap accurately, it would result in a histogram as on the left hand and if the images do not overlap, the histogram would be dispersed as in the histogram on the right hand side.

Mutual Information technique is born out of Information Theory. Intensities of the pixels at each coordinate of the source and the target images are utilized for the registration. Mutual Information method uses the concept of entropy, i.e., it relies on the amount of data that is known about images A and B and the unknown information that can be gathered from the known data.

The basic concept is to maximize the mutual information. The images are to be set in such a manner that MI of the two images is maximum, which can be obtained by minimizing the entropy.

Pluim et al [10] has used the concept of Shannon entropy. They have defined mutual information of two images A and $\mathrm{B}$ as:

$\mathrm{I}(\mathrm{A}, \mathrm{B})=\mathrm{H}(\mathrm{B})-\mathrm{H}(\mathrm{B} \mid \mathrm{A})$,

where $\mathrm{H}(\mathrm{B})$ is the Shannon entropy of the imageB, computedon the probability distribution piof the grey values.

$$
H(B)=-\sum_{i=1}^{n} p_{i} \log _{2} p_{i}
$$

Once $\mathrm{H}(\mathrm{B})$ is computed, $\mathrm{H}(\mathrm{B} \mid \mathrm{A})$ can be calculated where $\mathrm{H}(\mathrm{B} \mid \mathrm{A})$ is the information that can be gathered from $\mathrm{A}$ after knowing about $\mathrm{B}$.

Williams have given the following equation for Mutual Information I(A, B) [11]. 


$$
I(A, B)=\sum_{a, b} p_{A, B}(a, b) \log \frac{p_{A, B}(a, b)}{p_{A}(a) \cdot p_{B}(b)}
$$

Where, $\mathrm{pA}, \mathrm{B}(\mathrm{a}, \mathrm{b})$ is the joint probability mass function, obtained from joint intensity histogram.

$\mathrm{pA}(\mathrm{a})$ and $\mathrm{pB}(\mathrm{b})$ are the marginal probability mass functions. The marginal probability is high when the corresponding parts in the images are aligned.

When working with images, the functional form of the probability mass functioncannot be accessed accurately, so we need normalized histograms of the intensity values for each image.

Studholme et al. proposed a normalization formula of mutual information [12].

$$
I(A, B)=\operatorname{NMI}(A, B)=\frac{H(A)+H(B)}{H(A, B)}
$$

MI is calculated using the following formula:

$$
I\left(A, B^{\prime}, T_{\vec{\alpha}}\right)=\sum_{a, b^{\prime}} p_{A, B^{\prime},}\left(a, b^{\prime}\right) \log \frac{p_{A \cdot B^{\prime}}\left(a, b^{\prime}\right)}{p_{A}(a) \cdot p_{B^{\prime}}\left(b^{\prime}\right)}
$$

As discussed earlier, image registration uses transformation. Let image B is getting transformed to align itself with image A. Then the transformation equation can be written as [13]:

Where image B transforms to image B'.

$$
\mathrm{B}^{\prime}=\mathrm{T}_{\vec{\alpha}}(\mathrm{B})
$$

And the transformation is calculated using the following equations:

$$
\mathrm{T}_{\vec{\alpha}_{\text {reg }}}=\arg \max / \min \mathrm{I}\left(\mathrm{A}, \mathrm{B}^{\prime}, \mathrm{T}_{\vec{\alpha}}\right)
$$

MI is calculated and transformation is repeated until the algorithm reaches the maximum MI, i.e., best possible alignment fit of the two images.Mutual information method is a very costly method and bears the shortcomings of interpolation.

\section{Conclusion:-}

There are various techniques of rigid image registration. Every technique has its own shortcomings. The main idea of any transformation is to detect a transformation relationship between the supposedly corresponding coordinate points of the two images. The transformation is calculated and applied to the target image repeatedly till the images are properly aligned.

Once the images ae properly aligned, medical science can exploit the information gathered from the registered images to further diagnose the body part, especially the brain.

\section{References:-}

1. Alpert, N.M., Bradshaw, J.F., Kennedy, D and Correia J.A., 1990, 'The Principal Axes Transformation - A Method for Image Registration', The Journal of Nuclear Medicine, vol. 31, no. 10, pp. 1717-1722.

2. Kostelec, P.J. and Periaswamy, S., 2003, 'Image Registration for MRI', Modern Signal Processing, MSRI Publications, vol. 46, pp. 161-184.

3. Schormann,T. and Zilles, K., 1997, 'Limitations of the Principal-Axes Theory ', IEEE Transactions on medical imaging, Vol. 16, No. 6, pp. 942-947. 
4. Zitova,B. and Flusser, J., 2003, 'Image registration methods: a survey', Image and Vision Computing, Elsevier, vol. 21, pp. 977-1000.

5. Saxena, S. and Singh, R.K., 2014, 'A Survey of Recent and Classical Image Registration Methods', International Journal of Signal Processing, Image Processing and Pattern Recognition, vol. 7, no. 4, pp. 157176.

6. Tang, L. and and Hamarneh, G., 2013, 'Medical Image Registration: A Review (Chapter 22)', Medical Imaging: Technology and Applications, pp. 619-660.

7. Hill, D.L.G., Batchelor, P.G., Holden, M., and Hawkes, D.J., 2001, 'Medical image registration', Physics in Medicine and Biology, Institute of Physics Publishing, vol. 46, pp. R1-R45.

8. Woods, R.P., Mazziotta, J.C., and Cherry, S.R, 1993, 'MRI-PET Registration with Automated Algorithm', Journal of Computer Assisted Tomography, vol. 17, no. 4, pp. 536-546.

9. Hill, D.L.G., Hawkes, D.J., Harrison, N.A., and Ruff, C.F., 1993, 'A Strategy for Automated Multimodality Image Registration Incorporating Anatomical Knowledge and Imager Characteristics', IPMI '93 Proceedings of the 13th International Conference on Information Processing in Medical Imaging, pp. 182-196.

10. Pluim, J.P.W., Maintz, J.B.A. and Viergever, M.A., 2003,'Mutual information based registration of medicalimages: a survey', IEEE Transactions on Medical Imaging.

11. Williams, C., 2004, 'Rigid-Body Image Registration using MutualInformation', http://www4.ncsu.edu/ cmwilli5/BME231_Project_Report/Project_Report.pdf

12. Studholme, C., Hill, D.L.G., and Hawkes,D.J., 1999, 'An overlapinvariant entropy measure of 3D medical image alignment', Pattern Recognition, vol. 32, no. 1, pp. 71-86.

13. Gupta, N. and Garg, N., 2014, Review on Different Techniques of Image Registration, International Journal Of Engineering And Computer Science, vol. 3, no. 3, pp. 5093-5097. 